\title{
Reconstruction and Repairment With Mini-plate and Bone Graft for HIV Positive Patients of Giant Cell Tumor of Long Bone: Retrospective Analysis of a Single-center Experience
}

\section{Biao Xu}

Department of Orthopedics, Beijing Ditan Hospital, Capital Medical University

\section{Rui Ma}

Department of Orthopedics, Beijing Ditan Hospital, Capital Medical University

Qiang Zhang ( $\sim$ zhangq164@sina.com )

Department of Orthopedics, Beijing Ditan Hospital, Capital Medical University

\section{Chang-song Zhao}

Department of Orthopedics, Beijing Ditan Hospital, Capital Medical University

\section{Wen-sheng Zhang}

Department of Orthopedics, Beijing Ditan Hospital, Capital Medical University

Jie Wang

Department of Orthopedics, Beijing Ditan Hospital, Capital Medical University

\section{Research}

Keywords: HIV, Giant cell tumor, mini-plate, Bone morphologic repairment and reconstruction, Autogenous and allogeneic bone grafts

Posted Date: June 7th, 2021

DOI: https://doi.org/10.21203/rs.3.rs-554765/v1

License: (c) (i) This work is licensed under a Creative Commons Attribution 4.0 International License. Read Full License 


\section{Abstract}

Background: To evaluate the effect of reconstruction and repairment with mini-plate and bone graft for HIV positive patients of giant cell tumor of long bone.

Methods: This research retrospectively analyzed 12 HIV positive patients with giant cell tumor of long bone, 11 male and 1 female, with a age range 16 to 68 years old (43.5 years old on average) were included. There were 5 cases of proximal tibia $₫ 3$ cases of distal femur, 2 case of distal tibia, and 2 case of talus. From June 2012 to August 2020, curettage by ultrasonic scalpel were performed in all patients, combined with min- plate and bone graft treatment. All patients were followed up for 18-60 months. Limb function was evaluated by MSTS93 scoring system, and postoperative recurrence and distant metastasis, complications, MSTS93 score and fracture prognosis were observed.

Results: No local recurrence and pulmonary metastases was observed. After surgery, all the 12 patients showed good bone morphologic repair and reconstruction, good bone healing, good joint function, and no pathological fracture around the lesion. One case of giant cell tumor of proximal tibia showed mild articular surface collapse, and mild valgus deformity of knee joint, but good joint function. The MSTS 93

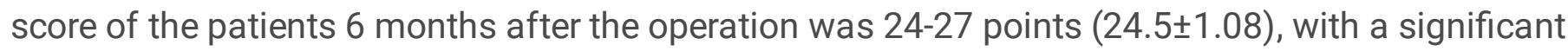
difference $(P<0.05)$.

Conclusion: Reconstruction and repairment with mini-plate and bone graft for HIV positive patients of giant cell tumor of long bone has achieved satisfactory results. The mini- plate takes up little space and is flexible for reconstruction and fixation, significantly reducing complications such as surgical site infection, preserving joint function and avoiding amputation. It is a safe and effective treatment method.

\section{Background}

Giant cell tumor of the bone (GCT) is a primary bone tumor, which accounts for $5-7 \%$ of all primary bone tumors and $20 \%$ of all benign bone tumors [1]. In China, its incidence was about $14-20 \%$, which was higher than $5-8 \%$ in other eastern countries[2]. GCT has unpredictable biological behavior with potential invasion, local recurrence, and low probability of distant metastasis [3-5], which tends to occur in people aged 20-40 years and female incidence is slightly higher than the male. GCT often occurs in the metaepiphyseal area of the limbs, especially in the distal femur and proximal tibia. GCT grow in an expansive manner and easily penetrate the cortex of the bone or even cause pathological fracture. According to literature reports, the incidence of pathological fractures is about $9 \% \sim 30 \%$ [6-9]. Pathological fractures are thought to be the result of more aggressive tumors, with a higher risk of recurrence and metastasis. Expanded intralesional curettage and segmental resection are two common surgical methods for pathologic fractures associated with GCT. The corresponding reconstruction methods include structural bone grafting, bone cement filling, bone plate fixation, external fixator, artificial joint replacement and allograft joint replacement [8-10]. 
GCT is a kind of osteolytic tumor, mononuclear cells and osteoclasts multiple nuclear cells as the main component. Acquired immune deficiency syndrome (AIDS) arises from human immunodeficiency virus (HIV) infection. HIV infection leads to a decrease of the count of CD4 T-cell, and the dysfunction of the macrophages and monocytes. It finally leads to immunodeficiency[11-12]. The question we are interested in is: Are HIV patients with GCT more likely to relapse and metastasize after pathological fracture? How are these patients treated with surgical intervention? However, at present, few literatures reported about HIV positive patients with GCT with pathological fracture and there are no effective treatments of this disease currently.

Conventional tumor curettage is performed with conventional steel plates, or even multiple steel plates, and some with bone cement. The disadvantages of this method are that it occupies a large space, has extensive soft tissue dissection, is not easy to heal the incision, has many complications, serious bone damage and weak cortex can not be fixed, and only large excision prosthesis replacement can not achieve the purpose of retaining the joint.There needs to be a way to repair the bone shape to provide some support and fixation for the bone graft, while preserving the joint around the tumor.Therefore, we studied the application of this mini- plate in combination with autologous and allogeneic bone grafts for bone morphologic repair and reconstruction, to carry out limb preservation treatment, and observe the efficacy after the operation, which has not been reported at present.

\section{Methods}

This study was reviewed and approved by the ethics committee of Department of Orthopedics, Beijing Ditan Hospital, Capital Medical University and conducted in accordance with the Declaration of Helsinki and its subsequent amendments. From June 2012 to August 2020, 12 HIV-positive patients with pathological fractures of giant cell tumor in limbs were selected from Beijing ditan hospital medical center for surgical treatment. The mean age of the 12 patients was 43.5 years (16-68 years). The ratio of men to women is 11 to 1 . Histamathological diagnosis of all cases was clear, except the patients with primary malignant giant cell tumor of bone, including 5 cases of proximal tibia, 3 cases of distal femur, 2 case of distal tibia, and 2 case of talus (Tables 1 ). All patients were followed up for 18-60 months after surgery, with an average of $31.24 \pm 11.84$ months. HIV infection was confirmed in all patients by enzymelinked immunosorbent assay (ELISA), with the lower limit of RNA detection $<20 \mathrm{copies} / \mathrm{ml}$. 
Table 1

Demographic and HIV -related details

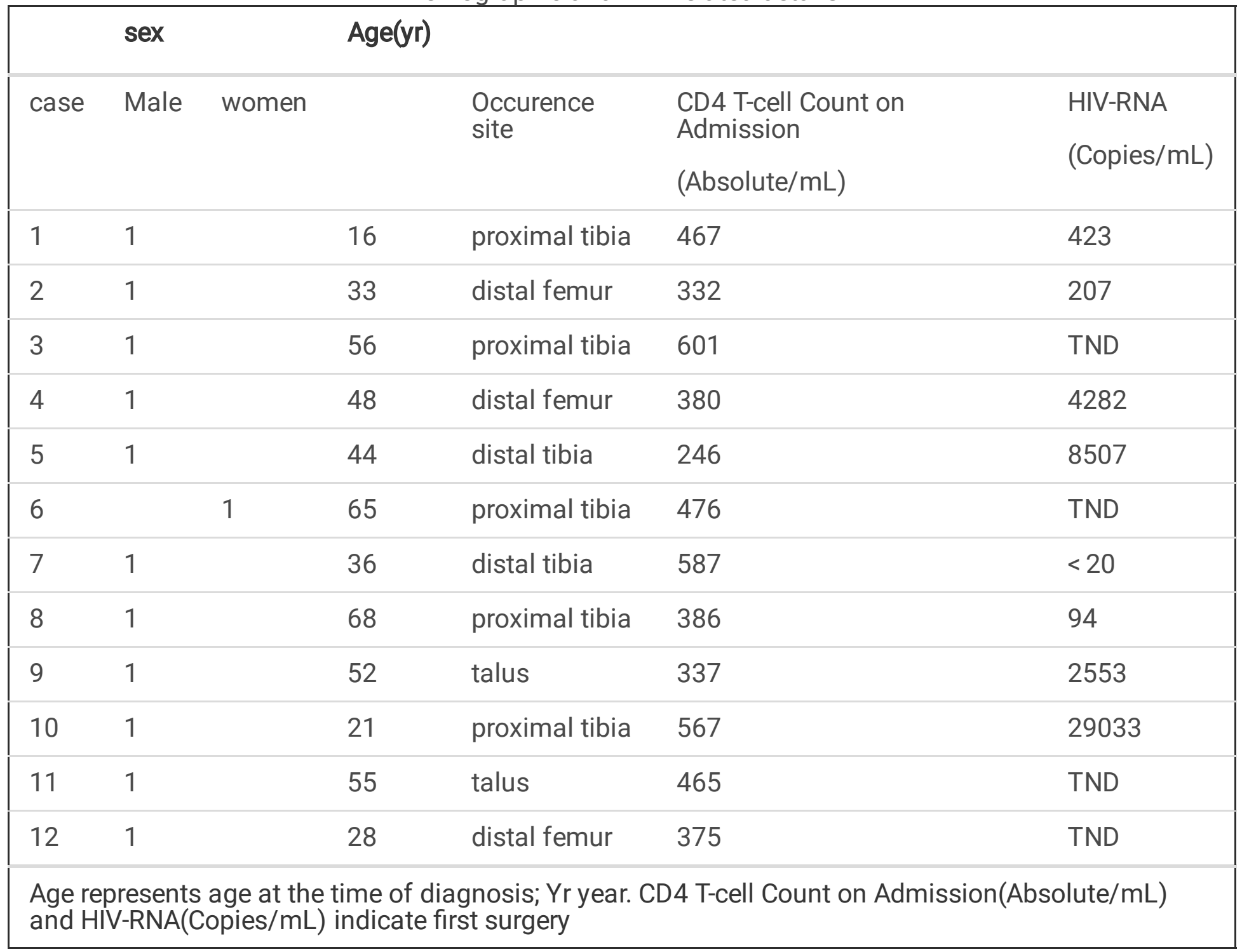

The blood CD4 cell count, HIV-RNA viral load, the application of antiviral drug HAART of the patients were collected from all the patients during the perioperative period. All tumors were treated with expanded intracapsular curettage. During the curettage process, the bone wall was ground and drilled with ultrasonic scalpel at high speed. The tumor cavity was inactivated with $10 \%$ hypertonic saline. The study program was approved by the ethics committee of Beijing ditan hospital and all participants' written consent was obtained before the study.

Ultrasonic treatment developed by SONOCA 300 SoringGmbH consists of three parts: main engine, handle and cutting tool, and cooling system(Fig. 1). The computer control of the electrical signals of ultrasonic frequency signal generator, a power amplifier amplification drive after ultrasonic transducer, ultrasonic transducer produces vibration under the action of electrical signals, the amplitude will drive the cutting tool work after amplitude amplification, working frequency for $(40 \pm 2) \mathrm{kHz}$, the implementation of automatic frequency tracking, equipped with $3 \mathrm{~mm}$ and $2 \mathrm{~mm}$ belt teeth and groove cutter, cut to fit 
different needs. The ultrasonic energy output of the cutter is set as $30 \%$. The handle has a self-cooling system, and the cutter can rotate slowly clockwise or counterclockwise as well as positive and negative rotation alternately, so as to increase the cutting ability.

All patients were first scraped with a spoon, the tumor wall was ground by ultrasonic scalpel, and then local chemotherapy with methotrexate and utogeous bone graft were used for treatment. Fully open the window when shaving, grinding carefully and repeatedly scratch tumor cavity wall and cavity bone crest, to the naked eye can see no tumor bone wall, reserved was not pay attention to keep the normal bone tumor invasion and epiphyseal plate, saline flushing repeatedly, local chemotherapy using methotrexate gelatin sponge, measuring the bone cavity size, iliac bone graft taken from the body cavity filling. According to different anatomical locations, the corresponding mini plate fixation screws were selected for internal fixation(Fig. 2).

In all patients, general radiography, chest X-ray, computed tomography (CT) and/or magnetic resonance imaging (MRI) were performed on more than one plane. In addition, all patients underwent needle puncture cytology and/or open biopsy. Postoperative score of limbs: the Musculoskeletal Tumor society (MSTS) 93 score was used for evaluation[13]. The upper limb function score included 6 items, including limb pain, activity function, acceptability, hand position, dexterity and lifting ability. Lower extremity function score included 6 items: limb pain, motor function, acceptability, support use, walking function and gait.

\section{Statistical analysis}

Gender, age, Occurence site, surgical treatment, postoperative complications, limb function, HIV related clinical data of all patients were collected and statistically analyzed. SPSS 18.0 software was used for statistical analysis. Measurement data were expressed as mean $\pm \mathrm{s}$, and $\mathrm{P}<0.05$ was considered statistically significant.

\section{Results}

No local recurrence and pulmonary metastases was observed. After surgery, all the 12 patients showed good bone morphologic repair and reconstruction, good bone healing, good joint function, and no pathological fracture around the lesion. One case of giant cell tumor of proximal tibia showed mild articular surface collapse, and mild valgus deformity of knee joint, but good joint function. The MSTS93 score of the patients 6 months after the operation was $24-27$ points $(24.5 \pm 1.08)$, with a significant difference.

For case No. 12( (Fig. 3-10), the patient, male, 28 years old, was admitted to the hospital by the outpatient department as "GCT and pathological fracture of the distal right tibia", mainly due to "pain and swelling of the right ankle caused by sprain downstairs, accompanied by limited movement for more than 2 months". 
Previous HIV infection history of 3 years, long-term oral HAART drug therapy, HCV positive, syphilis antibody positive, given penicillin treatment in the local hospital.

Physical examination: the right distal shank anterior tibial skin color is normal, no vein anger, skin temperature is slightly higher, local tenderness, no obvious radiation pain, the right distal tibia can touch the bone surface rough, the right distal tibia axial rattling pain is positive, the right ankle joint flexion and extension activity is limited, the right ankle dorsiflexion 10 degrees, plantar flexion 30 degrees, varus 20 degrees; The left ankle is dorsiflexion of 25 degrees and plantar flexion of 50 degrees.The dorsal foot artery beats well, and the toes feel normal blood transport. Auxiliary test: viral load TND.CD4T lymphocytes 375 cells/u.

$X$ - ray of the ankle joint showed a rounded, cystic destruction of the distal right tibia with expansion and thinning of the bone cortex(Fig. 3). CT results showed that the right tibia distal round cystic bone destruction, expansions, internal trabeculae disruption, bone cortical rupture Swelling of the surrounding soft tissue(Fig. 4). MRI plain scan of the ankle joint (October 20, 2017) : a mass at the distal end of the right tibia, about $29 \mathrm{~mm} \times 13 \mathrm{~mm} \times 24 \mathrm{~mm}$ abnormal Constant signal, TIWL shows equisignal with clear margin and visible bone destruction, which is considered as giant cell tumor of bone(Fig. 5)

Tumor curettage with ultrasonic knife, 95\% alcohol, local chemotherapy with methotrexate, bone graft fusion and internal fixation with micro plate and screw were performed(Fig. 6).

HE staining $(20 \mathrm{X}, 40 \mathrm{X})$ : A large number of giant cells and stromal cells were observed, accompanied by a small amount of focal hemorrhage and fibrous tissue hyperplasia ,Immunohistochemical staining (20X, 40X) : CD68 antigen positive(Fig. 7).

Postoperative anteroposterior and lateral X-ray examination of the ankle joint at 1 week, 1 year and 2 years: postoperative resection of the distal right tibia lesion with bone graft fusion plate Postoperative screw fixation. The bone graft was filled and fused well.Steel plate and screw fixed firmly, no fracture, bone nonunion, etc(Fig. 8). CT 1 year review of the ankle joint after surgery: resection of the distal right tibia lesion after bone graft fusion with plate and screw internal fixation. The bone graft was filled and fused well.Steel plate and screw fixed firmly, no fracture, bone nonunion, etc(Fig. 9).

The fixation was removed within 2 years after the operation. Follow-up: the right distal tibia lesion was removed by bone graft fusion plate and screw internal fixation, and the plate and screw were removed completely. Good bone graft fusion, good ankle movement, no cardiopulmonary metastasis(Fig. 10).

\section{Discussion}

HIV infection leads to a reduction of CD4 T-cell counts, dysfunction of the macrophages and monocytes, and thus finally leads to immunodeficiency, which is prone to various opportunistic infections and tumors[14]. Highly Active Antiretroviral Therapy (HAART) can inhibit virus replication, improve the immunity of patients, prolong the survival time of patients, and greatly improve the quality of life of 
patients [15-16].The related complications have gradually attracted people's attention, among which the research on bone tumor, osteoporosis and brittle fracture has gradually become a hot topic in recent years [17-18].

Given that increased inflammation, T-cell activation, and monocyte activation contribute to the pathogenesis of many HIV-related comorbidities [19]. We hypothesized that the pathological fracture of HIV with GCT may be related to the decreased immunity of the normal host, long-term chronic inflammatory stimulation, $T$ cell activation and abnormal activation of mononuclear macrophages. GCT is a kind of osteolytic tumor, mononuclear cells and osteoclasts multiple nuclear cells as the main component. Chronic inflammation is a well-established risk factor for bone loss. Bone resorption occurs through osteoclasts, highly specialized cells formed from the fusion of the origin of monocyte/macrophage cells [20-21]. In inflammatory conditions, RANKL is also expressed by activated T cells and B-cells, resulting in increased osteoclast activity and bone loss, which may lead to pathological fractures.

GCT are characterized by invasiveness, relapse, and pathological fracture. Since GCT is mostly located at the bone end and has the imaging characteristics of osteolytic destruction, eccentricity and expansion, it is not difficult to diagnose GCT with pathological fractures for the most part [22]. GCT of the long bone are relatively low in invasiveness. If the tumor size is small and the bone damage is light, the lesions can be simply scraped and transplanted without fixation to achieve stability. However, if the lesion range is wide, the bone cortex becomes thin due to severe erosion, and the biomechanical strength is reduced or even pathological fracture is complicated, it is particularly important to choose the appropriate method to fix the fracture.

In the early stage, benign bone tumors were mostly treated with external fixation stents [23]. With the development of internal fixators, intramedullary nails and steel plates are used more and more frequently. Intramedullary nails belong to axial fixation, and multi-center studies have found that the effect of intramedullary fixation is better [24]. At present, the miniature locking bone plate makes the fixation system more perfect as it has good fit, strong plasticity and easy anatomical fixation, does not take up excess space, and stabilizes the fracture end to promote fracture healing. In this group of cases, the miniature locking bone plate was used to bridge the lesion bone defect area to provide sufficient fixation strength and promote the normal healing of fractures. There was no loosening or displacement of internal fixator, and it was relatively easy to operate the technology of bridging locking plate on both sides of the fracture end after the removal of the lesion and bone grafting.

Most benign bone tumors had good prognosis and low recurrence rate after bone grafting. Reducing postoperative recurrence is one of the most concerned problems in the clinical treatment of benign bone tumors [25]. At present, many scholars try to use adjuvant therapy, such as carbonic acid, liquid nitrogen, bone cement treatment in order to reduce the recurrence rate, but did not find that adjuvant therapy can effectively reduce the local recurrence rate. Blackley et al. [26] reported that 59 cases of GCT were treated with high-speed grinding drill assisted curettage, with an average follow-up of 80 months and a 
recurrence rate of $12 \%$. The postoperative recurrence rate of patients in our study was low, which may be related to the routine use of Ultrasonic scalpel during the operation. Ultrasonic scalpel can reduce the difficulty of surgery, shorten the operation time, remove the bone crest tumor cells that cannot be scraped by conventional curettles, achieve the effect of edge resection, and ensure the thoroughness of surgery. Cutting and tumor tissue damage effect more ideal, make the surgery more safe and cutting temperature is low, is $70{ }^{\circ} \mathrm{C} \sim 80^{\circ} \mathrm{C}$, to destroy the cancer cells, further reduce the recurrence of the tumor.

All patients received highly effective retrovirus drug therapy, and the viral load control was not ideal, with an average of 87323 copies $/ \mathrm{ml}$. Although no tumor recurrence or distant metastasis occurred in all patients, 4 patients of 14 had surgical site infection, with an incidence rate of $28.5 \%$, which may be related to low immunity and HIV-RNA viremia. It has been confirmed that T-lymphocyte-mediated cellular immunity plays a major role in tumor immunity[27]. The T lymphocyte subsets of normal body coordinate with each other and play an effective role in cellular immunity. The results of this study showed that the T lymphocytes of HIV-positive patients with GCT decreased as a whole and the CD4+ / CD8 + ratio decreased, suggesting that the immune function of the body was further impaired, creating conditions for surgical site infection [28].

The MSTS93 score system was used to evaluate the postoperative score improvement of each patient. The MSTS93 score of the patients 6 months after the operation was $24-27$ points ( $24.5 \pm 1.08)$, with a significant difference $(P<0.05)$, which indicated that all patients were satisfied with the short-term postoperative effect.

This study also has some limitations:(1) the sample size was not enough and the follow-up time was short, so the clinical curative effect remains to be studied further; (2)MSTS93 scoring system can provide an evaluation scale for patients after limb surgery, but this system is affected by subjective factors, including patient cooperation degree, patient cognitive ability, and physician clinical experience, and cannot achieve objective evaluation. But long-term efficacy remains to be seen.

\section{Conclusions}

Ultrasonic scalpel combined with miniature locking bone plate and autogeous bone graft treatment showed satisfactory results in HIV positive patients with giant cell tumor of long bone. And it is a safe and efficient treatment. In the surgical design, the nature, scope and fracture damage of the lesion should be taken into full consideration. During the operation, the lesion should be thoroughly scraped, the tumor wall should be polished with ultrasonicscalpel, and bone grafting should be put under pressure. At the same time, appropriate miniature locking bone plate fixation is helpful for fracture healing, which is an effective method to improve the clinical efficacy.

\section{Abbreviations}


HIV: human immunodeficiency virus; MSTS: Musculoskeletal Tumor society; GCT: Giant cell tumor of bone; enzyme-linked; ELISA: immunosorbent assay $\mathbb{} \mathrm{CT}$ : computed tomography $\mathbb{\mathrm { MRI }}$ : magnetic resonance imaging $\mathbb{H}$ AART: Highly Active Antiretroviral Therapy.

\section{Declarations}

\section{Acknowledgements}

Not applicable.

\section{Funding}

We acknowledge financial support by Capital's Funds for Health Improvement and Research (No.2018-22174), Scientific Research Common Program of Beijing Municipal Commission of Education (KM201810025029) and Beijing Municipal Science \& Technology Commission (No. Z191100006619060).

\section{Availability of data and materials}

All data generated or analyzed during this study are included in this published article and its supplementary information files.

\section{Ethics approval and consent to participate}

This retrospective study was approved by the ethical committee of Beijing Ditan Hospital, Capital Medical University and performed in accordance with the ethical standards of the 1964 Declaration of Helsinki. And all participants signed informed consent for publication.

\section{Consent for publication}

Not applicable.

\section{Competing interests}

The authors declare that they have no competing interests.

\section{Authors' contributions}

Biao Xu, Rui Ma and Qiang Zhang conceived the study. Biao Xu wrote the original manuscript. Qiang Zhang performed the surgery. Biao Xu, Rui Ma and Qiang Zhang checked the data and revised the manuscript. Chang-song Zhao, Wen-sheng Zhang and Jie Wang contributed in collecting and analyzing the patient data and following them up. All authors read and approved the final manuscript.

\section{References}


1. Unni KK, Inwards CY. Dahlin's Bone Tumors: General Aspects and Data on 10,165 Cases. Rochester, MN: Lippincott Williams \& Wilkins (2010).

2. Niu X, Zhang Q, Hao L, Ding Y, Li Y, Xu H, et al. Giant cell tumor of the extremity: retrospective analysis of 621 Chinese patients from one institution. J Bone Joint Surg Am. (2012) 94:461-7. doi: 10.2106/JBJS.J.01922

3. Jeys LM, Suneja R, Chami G, Grimer RJ, Carter SR, Tillman RM. Impending fractures in giant cell tumours of the distal femur: incidence and outcome. Int Orthop. 2006;30:135-8.

4. Campanacci M, Baldini N, Boriani S, Sudanese A. Giant-cell tumor of bone. J Bone Joint Surg Am. 1987;69:106-14.

5. Muheremu A, Niu X. Pulmonary metastasis of giant cell tumor of bones. World J Surg Oncol. 2014;12:261.

6. Campanacci M, Baldini N, Boriani S, et al. Giant cell tumor of bone. J Bone joint Surg Am, 1987, 69(1): 106-14.

7. Ghert MA, Rizzo M, Harrelson JM, et al. Giant-cell tumor of the appendicular skeleton. Clin Orthop Relat Res, 2002 Jul;(400):201-10.

8. Turcotte RE, Wunder JS, Isler MH, et al. Giant cell tumor of long bone: a Canadian Sarcoma Group study. Clin Orthop Relat Res, 2002 Apr;(397):248-58.

9. Kivioja $\mathrm{AH}$, Blomgvist $\mathrm{C}$, Hietaniemi $\mathrm{K}$, et al. Cement is recommended in intralesional surgery of giant cell tumors: a Scandinavian Sarcoma Group study of 294 patients followed for a median time of 5 years. Acta Orthop, 2008 Feb;79(1):86-93.

10. LewisVO, Wei A, Mendoza T, et al. Argon beam coagulation as an adjuvant for local control of giant cell tumor. Clin Orthop Relat Res. 2007 Jan; 454:192-7.

11. Zapata HJ and Shaw AC. Aging of the human innate immune system in HIV infection. Curr Opin Immunol 2014; 29: 127-136.

12. Abalo A, Patassi A, James YE, Walla A, Sangare A and Dossim A. Risk factors for surgical wound infection in HIV-positive patients undergoing surgery for orthopaedic trauma. J Orthop Surg (Hong Kong) 2010; 18: 224-227.

13. Enneking WF, Dunham W, Gehhardt M C , et al. A system for the functional evaluation of reconstructive procedures after surgical treatment of tumors of the musculoskeletal system [M J. IIClassic Papers in Orthopaedics. London: Springer , 20 14: 241-246.

14. Sobottke R, Zarghooni K, Krengel M, Delank S, Seifert H, Fatkenheuer G,Ernestus I, Kallicke T, Frangen T, Arasteh K, Oette M and Eysel P. Treatment of spondylodiscitis in human immunodeficiency virusinfected patients: a comparison of conservative and operative therapy. Spine (Phila Pa 1976) 2009; 34: E452-458.

15. Kaplan-Lewis E, Aberg JA, Lee M. Aging with HIV in the ART era. Semin Diagn Pathol. 2017 Jul;34(4):384-397. doi: 10.1053/j. 
16. Lifson AR, Grandits GA, Gardner EM,et al. Quality of life assessment among HIV-positive persons entering the INSIGHT Strategic Timing of AntiRetroviral Treatment (START) trial. HIV Med. 2015 Apr;16 Suppl 1:88-96. doi: 10.1111/hiv.12237.

17. Gonciulea A, Wang R, Althoff KN, et al. An increased rate of fracture occurs a decade earlier in HIV+ compared with HIV- men. AIDS. 2017 Jun 19;31(10):1435-1443. doi:

10.1097/QAD.0000000000001493.

18. Krikke M, Klomberg RCW, Veer E, et al. Osteoporosis and osteopenia are not associated with T-cell activation in older cART-treated HIV-infected patients. Neth J Med. 2017 May;75(4):138-144.

19. Ofotokun I, Mclntosh E and Weitzmann MN. HIV: inflammation and bone. Curr HIV/AIDS Rep 2012; 9: 16-25

20. O’Brien CA. Control of RANKL gene expression. Bone 2010; 46: 911-919.

21. Kawai T, Matsuyama T, Hosokawa Y, Makihira S, Seki M, Karimbux NY, Goncalves RB, Valverde $P$, Dibart S, Li YP, Miranda LA, Ernst CW, Izumi Y and Taubman MA. B and T lymphocytes are the primary sources of RANKL in the bone resorptive lesion of periodontal disease. Am J Pathol 2006; 169: 987-998.

22. Li X, Guo, W, Yang Y, et al. Surgical treatment for long ipads giant cell tumor of extremity with pathologic fracture. Journal of Peking University (Health Science). 2013 Oct 18th;45 (5): 745-51.

23. Qin buping, Qian zheng, Huang yonggang. The Application of External Fixture in Treatment of limb pathologic Fracture with Bone Osteoma. Journal of Practical Orthopedics, 2001,7 (6): 418-9.

24. Ippolito E, Bray EW, Corsi A, et al. Natural history and treatment of fibrous dysplasia of bone: a multicenter clinicopathologic study promoted by the European Pediatric Orthopaedic Society. J Pediatr Orthop B. 2003 May ;12(3) : 155-77.

25. Shih HN, Shih KY, Cheng CY, et al. Reconstructing humerus defects after tumor resection using an intramedullary cortical allograft strut, Chang Gung Med J, 2002, Oct; 25 (10): 656-63.

26. Blackley HR, Wunder JS, Davis AM, et al. Treatment of giant-cell tumors of long bones with curettage and bone-grafting.J Bone Joint Surg Am. 1999 Jun;81(6):811-20.

27. Ma huiqun, Tian Zhongwei, Feng Jie, et al. Effects of PULEKANG on the immunological function in patients with HIV/AIDS. Journal of Xi 'an Jiaotong University (Medical Sciences), 2004,25 (6): $608-9$.

28. Chen Weilie, Yuan Xiaozhen, Tang yangbo, et al. Comparison of T lymphocyte subpopulation counts in people living with HIV/ AIDS and tumor patients. Chin J AIDS/ STD, 2003, 9(6): 338-40.

\section{Figures}


a

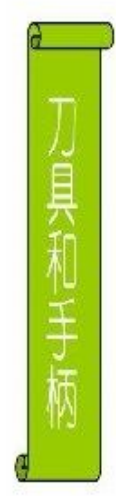

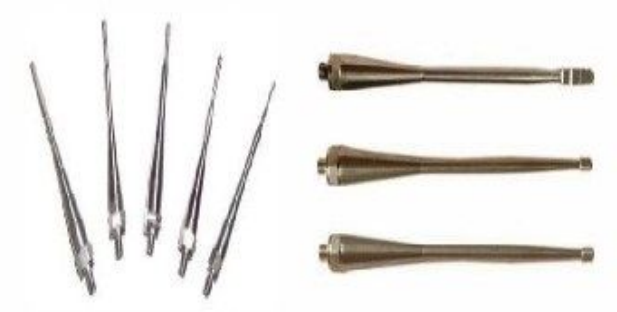

F放手材具

b

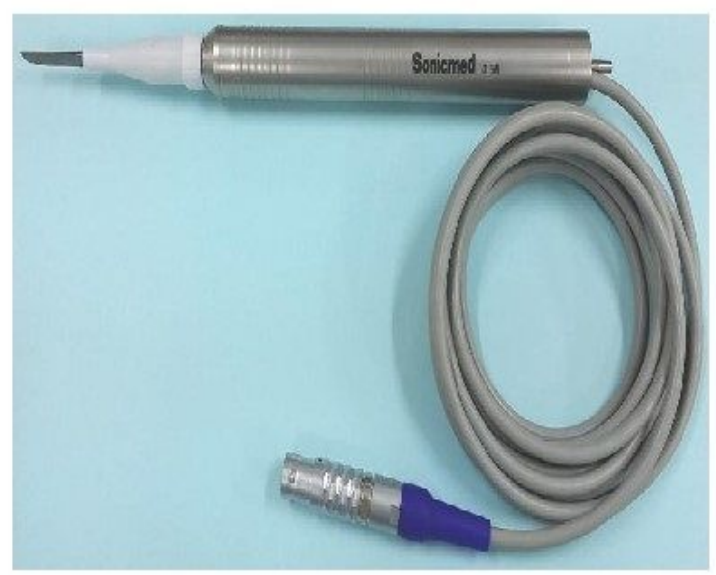

d

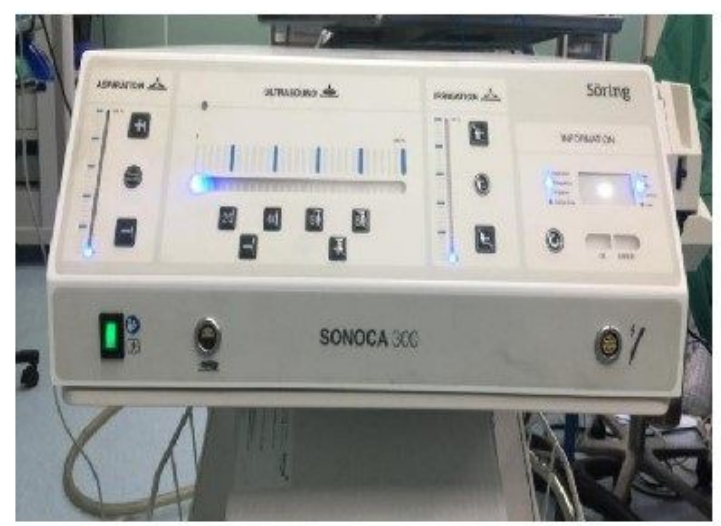

\section{Figure 1}

SONOCA 300 SoringGmbH:Surgical tools(a) ,Cut the bone handle(b),Grind bone handle(c), main engine and cooling system(d). 


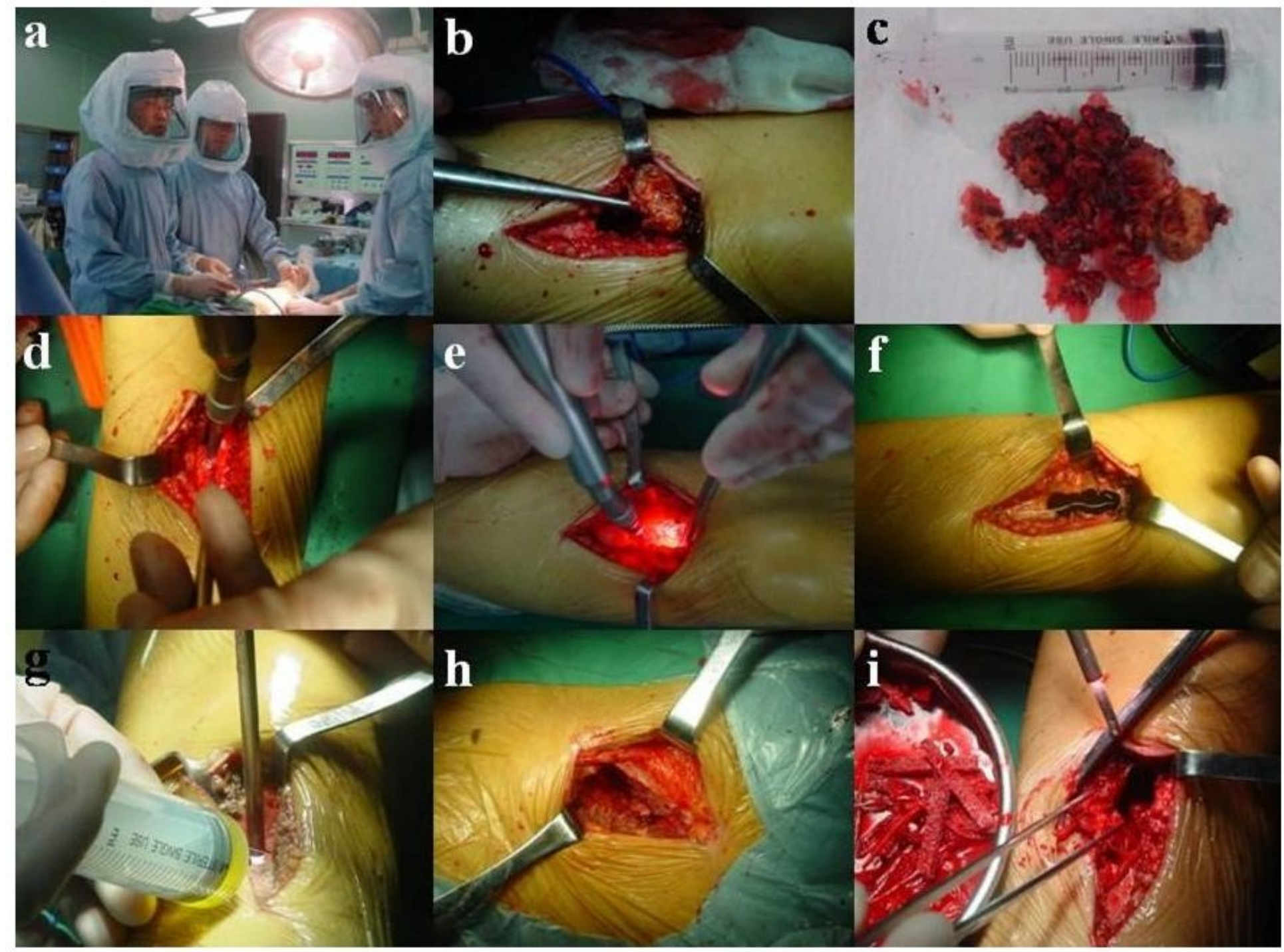

\section{Figure 2}

During the operation, the lesion was scraped and the bone cavity was polished with ultrasonic knife(a-f), the polished inner wall of the bone cavity was burned with anhydrous alcohol, and local methotrexate chemotherapy was performed(g), Autogenous iliac crest or allograft(h, i) 


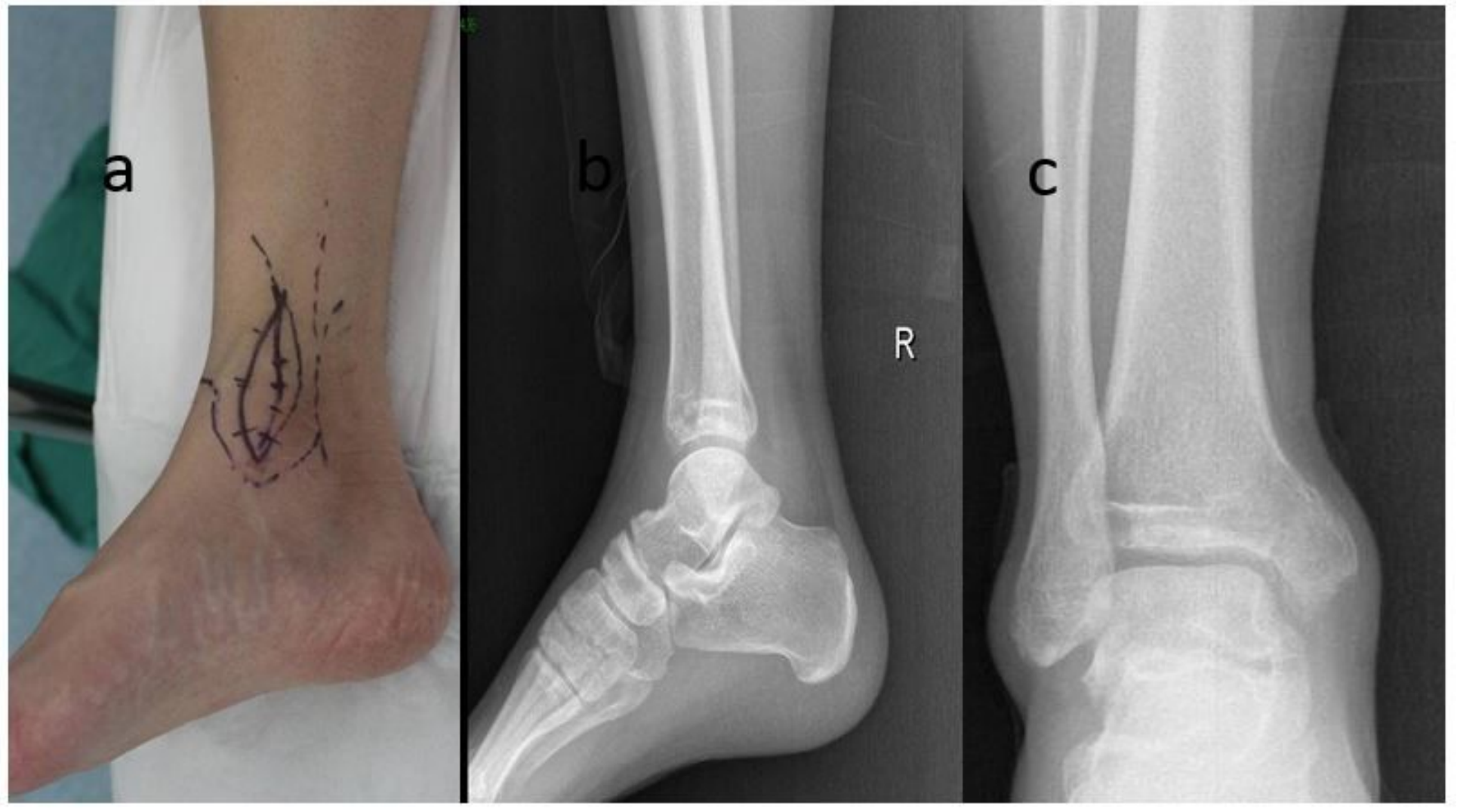

Figure 3

Preoperative general image (a), $\mathrm{X}$ - ray of the ankle joint showed a rounded, cystic destruction of the distal right tibia with expansion and thinning of the bone cortex $(b, c)$

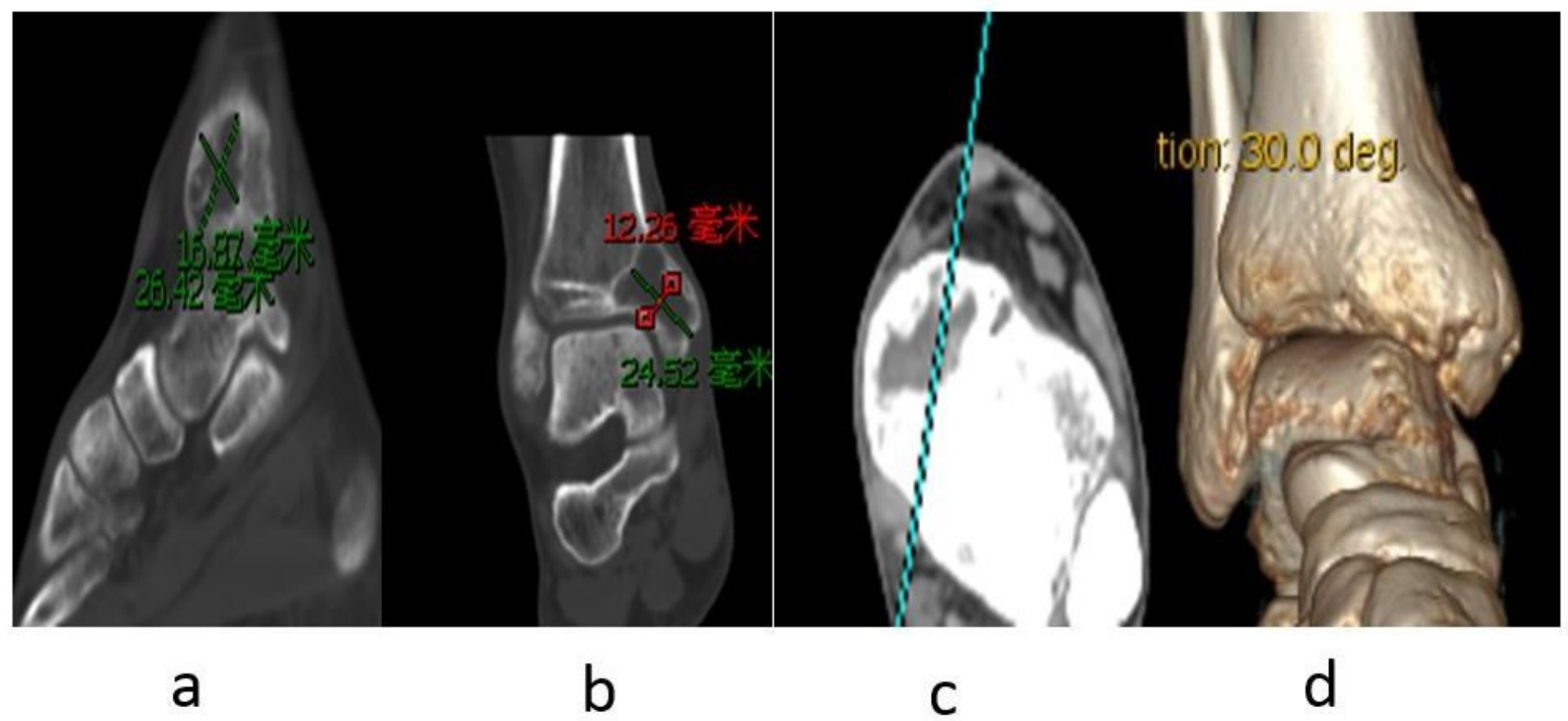

Figure 4 
CT results showed that the right tibia distal round cystic bone destruction, expansions, internal trabeculae disruption, bone cortical rupture Swelling of the surrounding soft tissue(a-d)

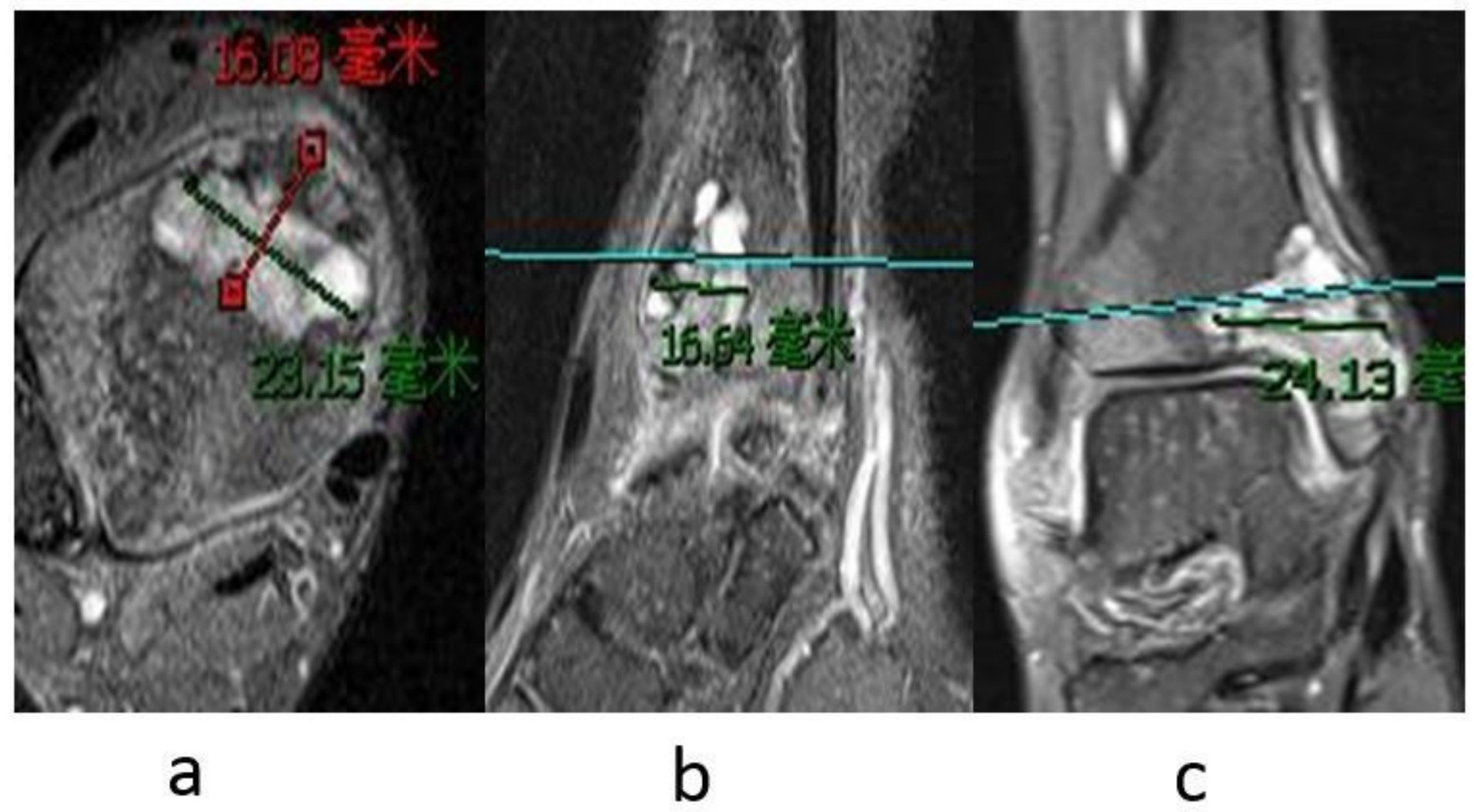

\section{Figure 5}

MRI plain scan of the ankle joint (October 20,2017) : a mass at the distal end of the right tibia, about 29 $\mathrm{mm} \times 13 \mathrm{~mm} \times 24 \mathrm{~mm}$ abnormal Constant signal, TIWL shows equisignal with clear margin and visible bone destruction, which is considered as giant cell tumor of bone(a-c) 


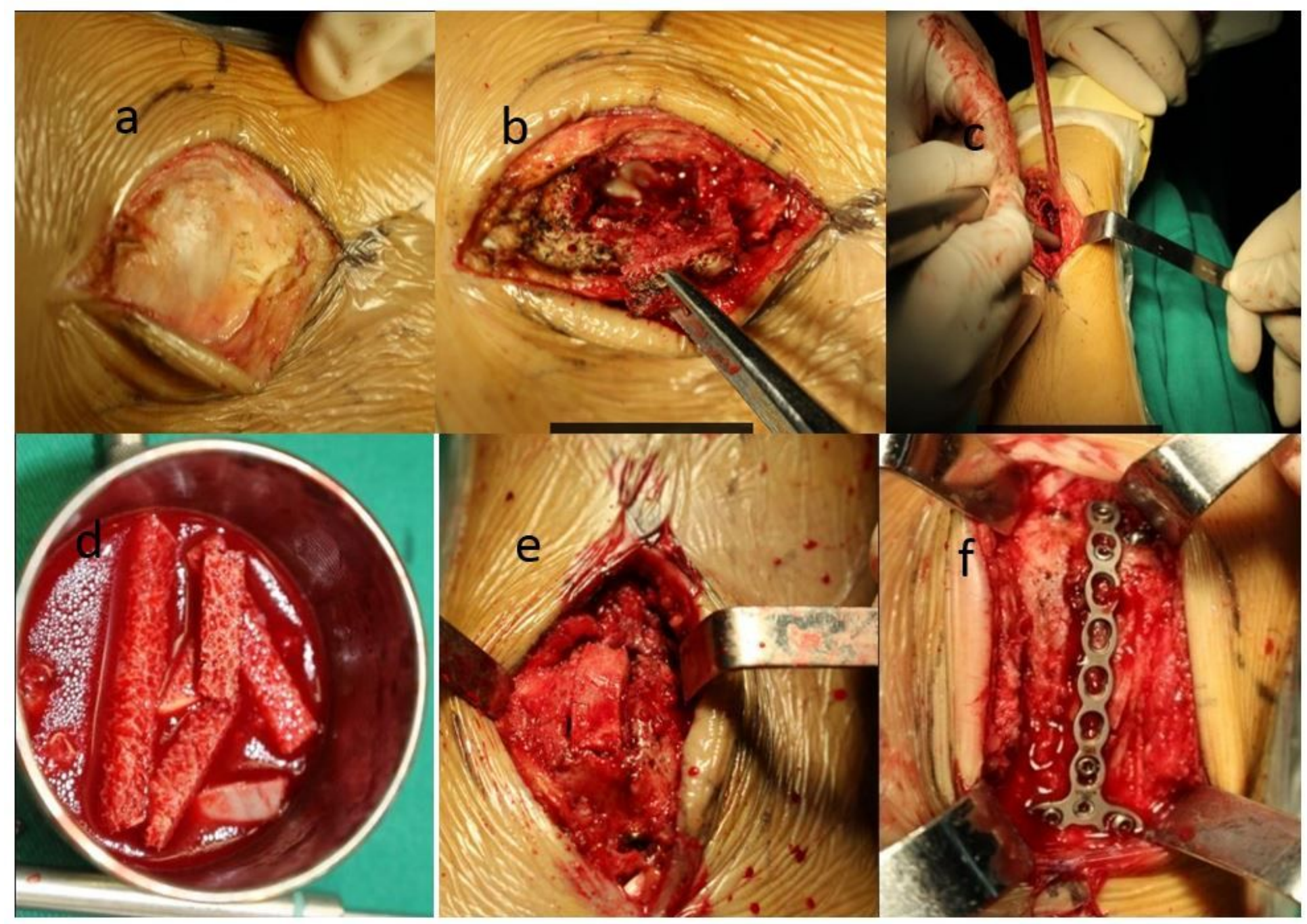

Figure 6

Tumor curettage with ultrasonic knife, 95\% alcohol, local chemotherapy with methotrexate(a-c), bone graft fusion $(d, e)$ and internal fixation with micro plate and screw(f) were performed 


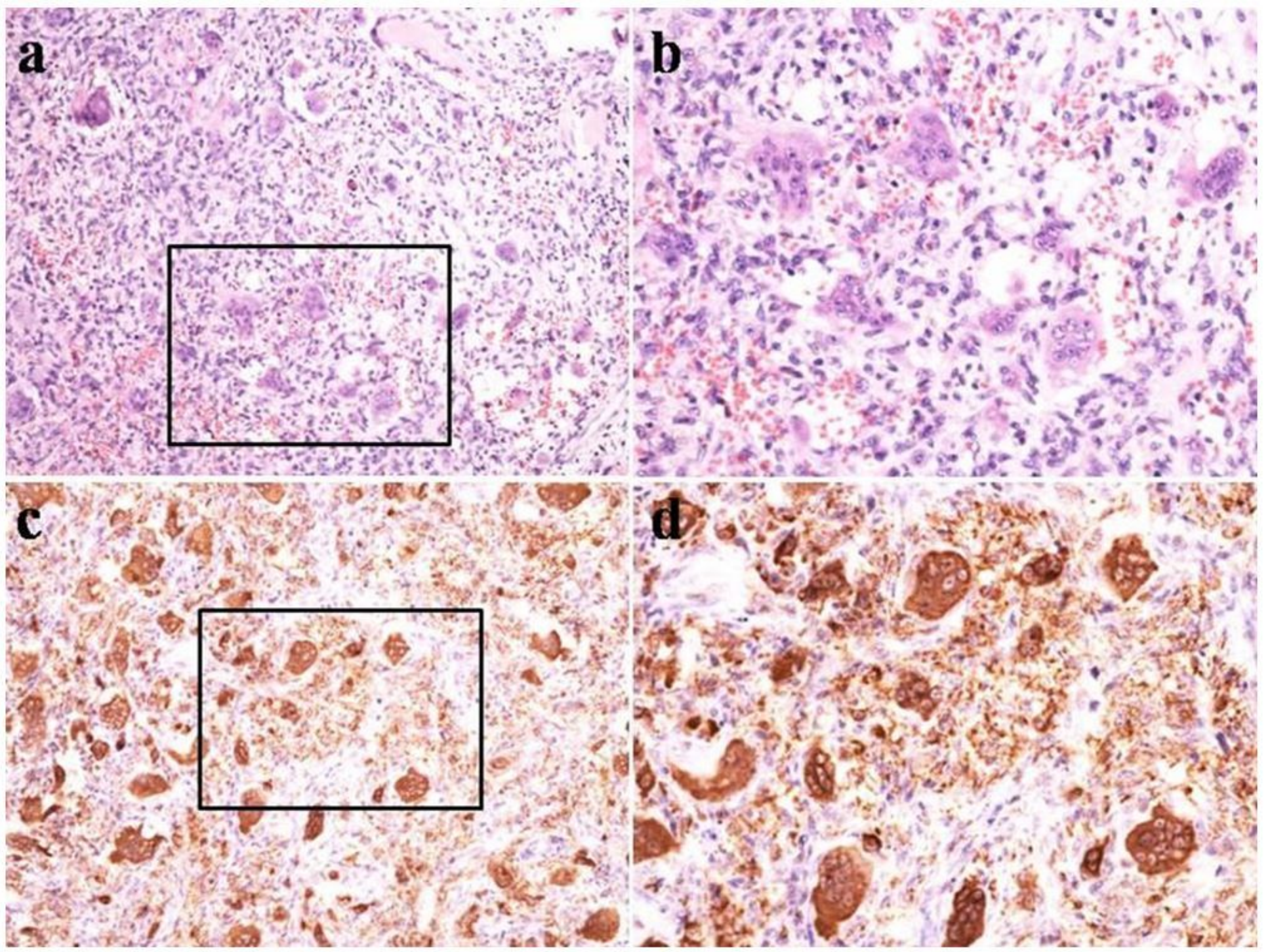

\section{Figure 7}

HE staining (20X,40X) : A large number of giant cells and stromal cells were observed, accompanied by a small amount of focal hemorrhage and fibrous tissue hyperplasia(a, b), Immunohistochemical staining (20X, 40X) : CD68 antigen positive(c, d) 


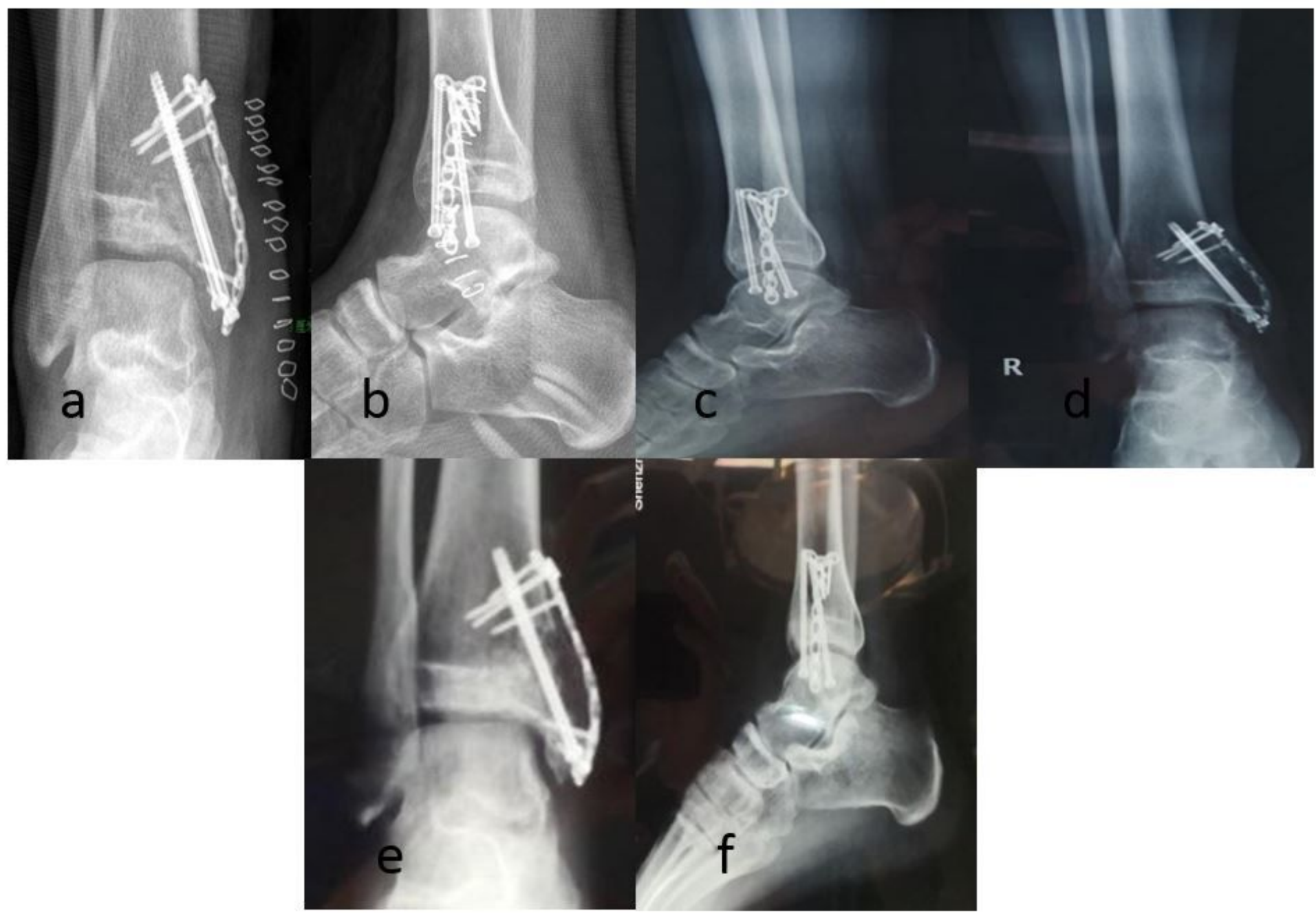

\section{Figure 8}

Postoperative anteroposterior and lateral X-ray examination of the ankle joint at 1 week(a, b), 1 year(c, d) and 2 years $(e, f)$ : postoperative resection of the distal right tibia lesion with bone graft fusion plate Postoperative screw fixation. The bone graft was filled and fused well. Steel plate and screw fixed firmly, no fracture, bone nonunion, etc

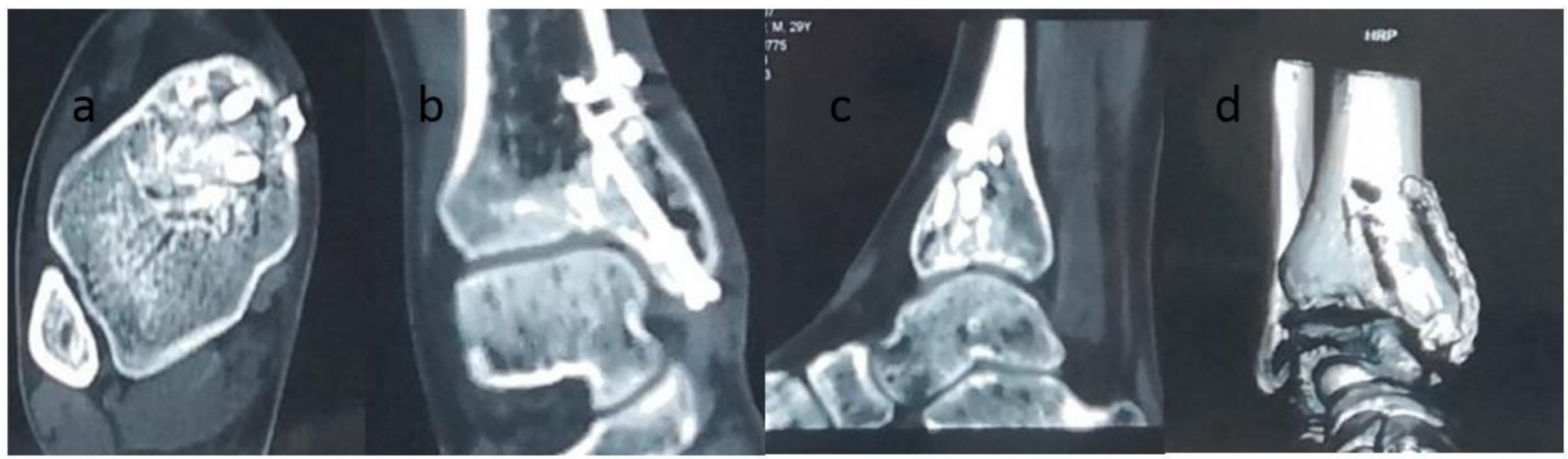

Figure 9 
CT 1 year review of the ankle joint after surgery(a-d): resection of the distal right tibia lesion after bone graft fusion with plate and screw internal fixation. The bone graft was filled and fused well. Steel plate and screw fixed firmly, no fracture, bone nonunion, etc

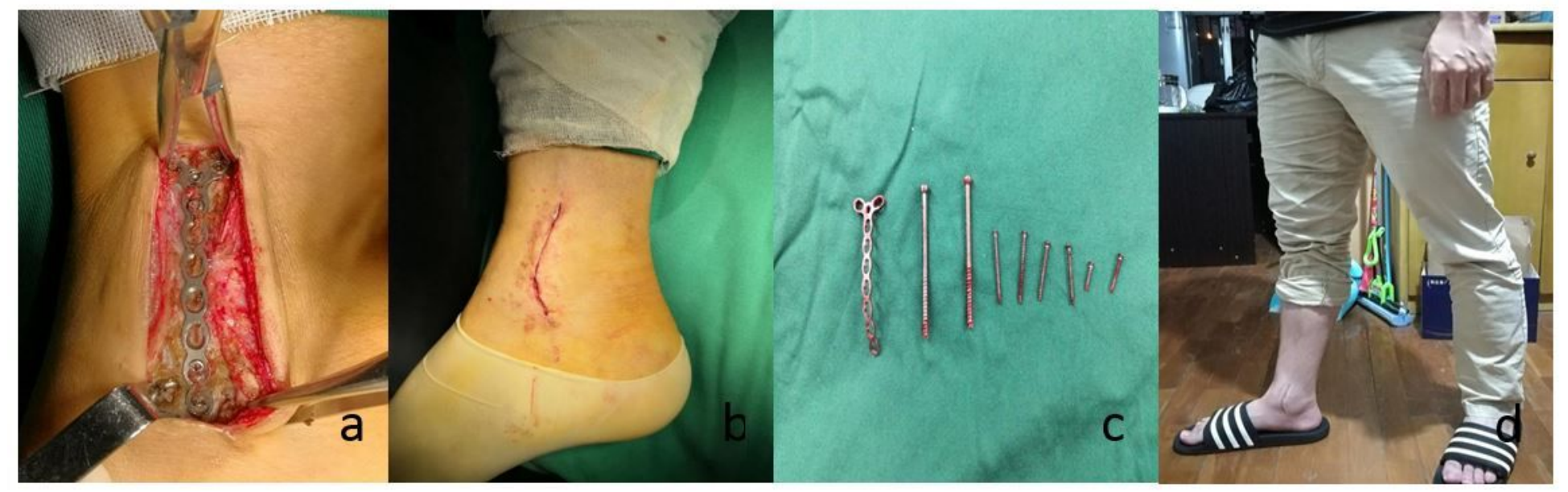

\section{Figure 10}

The fixation was removed within 2 years after the operation. Follow-up: the right distal tibia lesion was removed by bone graft fusion plate and screw internal fixation, and the plate and screw were removed completely(a-c). Good bone graft fusion, good ankle movement, no cardiopulmonary metastasis(d). 